\title{
ON THE REALIZATION OF HOMOLOGY CLASSES BY SUBMANIFOLDS
}

\author{
BY \\ HARUO SUZUKI
}

1. Introduction. In [1] $\mathrm{R}$. Thom defines when a cohomology class in a manifold is realizable for a closed subgroup of an orthogonal group. He also defines when a homology class is realizable by a submanifold. He then shows the following.

Let $m$ be the dimension of a compact orientable differentiable manifold $M$. Any cohomology class of dimension $1,2, m-6, m-5, \cdots, m$ is realizable for the orthogonal group $O(1)=1, O(2), O(m-6), O(m-5), \cdots, O(m)$ respectively. If a class $z \in H_{m-k}(M ; Z)$ can be realized by a submanifold, then the cohomology class $u \in H^{k}(M ; Z)$ which is dual to $z$ satisfies

$$
S t_{p}^{2 r(p-1)+1}(u)=0
$$

for all integers $r$ and all odd primes $p$. Here $S t_{p}^{2 r(p-1)+1}$ denotes the Steenrod reduced power [4] which operates on the cohomology group with integral coefficients $Z$. All homology classes with integral coefficients of compact orientable differentiable manifolds of dimension $<10$ are realizable by submanifolds.

We consider compact differentiable manifolds which are not necessarily orientable, and we ask whether the cup-products and the Steenrod squares [3] of realizable cohomology classes can be realized. It was stated by Thom [1] that cup-products of realizable classes are also realizable. We shall give another proof of this result (see $\S 3$ below). As for squares of realizable classes with integers modulo 2 as coefficients, we have the following result. Let $u$ be a cohomology class of dimension $n$ in a compact differentiable manifold of dimension $m+n$. If $u$ is realizable for the group $O(k) \subset O(n)(k \leqq n)$, then the cohomology class $S q^{k}(u)$ is also realizable (see $\S 5$ below).

The author thanks Professor R. Thom and E. Spanier for their valuable suggestions.

2. Preliminaries. In this section, we summarize the theory of Thom [1 and 2]. Let $\left.{ }^{1}\right) G$ be a closed subgroup of the orthogonal group $O(n)$. Let $S$ denote an $(n-1)$-sphere fiber space over a finite cell complex $K$ with the structural group $G$ and let $A$ be the mapping cylinder of the projection of $S$ onto $K$. $A$ becomes a fiber space over $K$ with a closed $n$-cell $b_{n}$ as fiber. Its projection is induced by that of $S$. We denote by $A^{\prime}$ the complement of $S$ in $A$ which is a fiber space over $K$ with fiber, an open $n$-cell $b_{n}$.

Received by the editors August 13, 1956 and, in revised form, October 19, 1956.

(1) If $G$ is a compact Lie group, then there is an integer $n$ such that $G$ is isomorphic to a subgroup of $O(n)$. 
Throughout this paper, (\&) will denote the group of integers or integers modulo 2. When dealing with the nonorientable case $\left({ }^{2}\right)$ (f) will denote only the group of integers modulo 2 .

By the theory of fiber spaces with open cells as fibers, we have a canonical isomorphism $\phi^{*}$ of the $r$ dimensional cohomology group $H^{r}(K$; ()$)$ onto the $r+n$ dimensional cohomology group $H^{r+n}\left(A^{\prime}\right.$; (S) $)=H^{r+n}\left(A, S\right.$; (S).$H^{n}(A, S$;(S) is the first nonvanishing cohomology group.

We denote by $S_{G, n}$ and $B_{G}$ the universal fiber space and the classifying space respectively, for $(n-1)$-sphere fiber spaces over finite cell complexes of bounded dimension with the structural group $G$ (see [5]). We may assume $B_{G}$ is a grassmann manifold. Let $A_{G, n}$ be the mapping cylinder of the projection of $S_{G, n}$ onto $B_{G}$ and let $A_{G, n}^{\prime}$ be the complement of $S_{G, n}$ in $A_{G, n}$. In particular, we denote $S_{O(n), n}, A_{O(n), n}$ and $A_{O(n), n}^{\prime}$ by $S_{O(n)}, A_{O(n)}$ and $A_{O(n)}^{\prime}$ for the sake of brevity.

Definition 1. Let $M(G, n)$ be the space which we get from $A_{G, n}$ by identifying its boundary $S_{G, n}$ to a point. We call the space $M(G, n)$ the cell complex corresponding to the subgroup $G$ of $O(n)$. We denote $M(O(n), n)$ simply by $M(O(n))$.

We have the natural isomorphisms of cohomology groups,

$$
H^{r}\left(A_{G, n}^{\prime} ; \text { (j) }\right)=H^{r}\left(A_{G, n}, S_{G, n} ; \text { (j) }\right)=H^{r}(M(G, n) \text {; (j) })
$$

where $r>0$. It is quite easy to see that $H^{0}\left(M(G, n)\right.$; $(\xi)=(\xi) \phi_{G, n}^{*}$ denotes the canonical isomorphism in the fiber structure $A_{G, n}^{\prime}$,

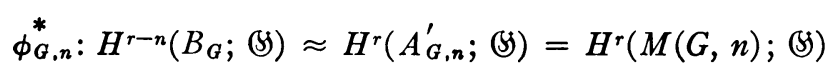

where $r \geqq n$. Therefore the cohomology groups $H^{r}(M(G, n)$; (5) of dimensions $r \geqq n$ are obtained by raising dimensions of cohomology groups of the classifying space $B_{G}$ by the integer $n . H^{n}(M(G, n)$; (s) is the first cohomology group which does not vanish in dimension $>0$. This group is generated by the class,

$$
U_{G, n}=\phi_{G, n}^{*}\left(1_{G}\right) \in H^{n}(M(G, n) ;(\$)
$$

where $1_{G}$ is the unit class of $H^{*}\left(B_{G}\right.$; (j). We call the class $U_{G, n}$ the fundamental class of $M(G, n)$. We denote $U_{O(n), n}$ simply by $U_{O(n)}$.

Definition 2. Let $A$ be a topological space and let $u$ be a class of

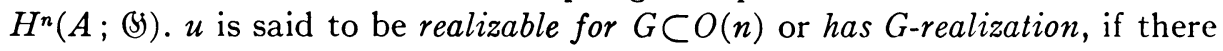
is a map $f: A \rightarrow M(G, n)$ such that $u=f^{*} U_{G, n}$.

Definition 3. Let $W$ be a submanifold of dimension $p$ in a compact differentiable manifold $V$ of dimension $m$ and of class $C^{\infty}$, where $p$ is an integer such that $m \geqq p \geqq 0$. The inclusion mapping $i: W \subset V$ induces a homomorphism $i_{*}$ of the homology group $H_{p}\left(W ;\right.$ (S) in to the homology group $H_{p}(V$; (S)).

${ }^{2}{ }^{2}$ Orientability means that of the fiber space $S$, therefore, of $A$. In this case, $G$ is connected. 
If a homology class $z$ of $H_{p}(W ;(\xi))$ is the image of the fundamental class of $W$, then we say that the class $z$ is realized by the submanifold $W$.

Now we state the following fundamental theorem [1, Chap. II]:

THEOREM 1. Let $V$ be a compact differentiable manifold of dimension $m$ and of class $C^{\infty}$, and let $n$ be an integer such that $m \geqq n \geqq 0$. A cohomology class $u \in H^{n}(V$; (S) is realizable for the group $G \subset O(n)$ if and only if the dual homology class $\left(^{(}\right) z$ of $u$ is realized by a submanifold $W$ of dimension $m-n$ and the fiber space consisting of normal vectors on the submanifold $W$ in $V$ has the group $G$ as its structural group.

Let $h$ be a classifying map of $K$ into $B_{G}$ for the fiber space $S$. Then it induces a fiber mapping $H$ of $A$ into $A_{G, n}$. Commutativity holds in the diagram,

$$
\begin{aligned}
A & \stackrel{H}{\rightarrow} A_{G, n} \\
p \downarrow & \stackrel{h}{\downarrow} p_{G, n} \\
K & \stackrel{h}{\rightarrow} B_{G}
\end{aligned}
$$

where $p$ and $p_{G, n}$ are the projections of the fiber structures $A$ and $A_{G, n}$ which are naturally induced by the projections $S \rightarrow K$ and $S_{G, n} \rightarrow B_{G}$. The commutativity of (2) induces that of the following diagram of homomorphisms of cohomology groups,

$$
\begin{aligned}
& H^{n}\left(A ^ { \prime } ; \text { (S) } \stackrel { H ^ { \prime * } } { \longleftarrow } H ^ { n } \left(A_{G, n}^{\prime} ;\right.\right. \text { (S) }
\end{aligned}
$$

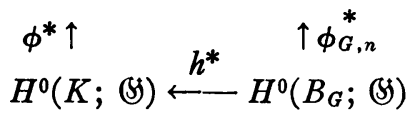

where $H^{\prime}$ is the mapping of $A^{\prime}$ into $A_{G, n}^{\prime}$ induced by $H$.

Obviously, the homomorphism $p^{*}$ induced by $p$ gives us an isomorphism of $H^{r}\left(K\right.$; (B) onto $H^{r}\left(A\right.$; (H). We put $\phi^{*}(1)=U_{n} \in H^{n}\left(A^{\prime}\right.$; (B) where 1 is the unit class of the cohomology ring $H^{*}(K$; (B) $)$. Let $\beta^{*}$ be the homomorphism of $H^{n}\left(A^{\prime}\right.$; (S) $=H^{n}\left(A, S\right.$; (j) into $H^{n}(A$; (S) induced by the inclusion map $\beta$ of $(A, 0)$ into $(A, S)$. The cohomology class $W_{n}=p^{*-1} \beta^{*} U_{n}$ in $H^{n}(K$; $(S)$ is called the fundamental characteristic class of the $(n-1)$-sphere fiber space $S$. In below, we shall denote $p^{*} W_{n}$ by the same symbol $W_{n}$.

The isomorphism $\phi^{*}$ has the following important property $\left({ }^{4}\right)$,

$$
\phi^{*}(x \cup y)=p^{*} x \cup \phi^{*} y
$$

(3) We remark the fact that if $V$ is orientable, then duality means the Poincare duality, i.e., the isomorphism of the homology group $H_{m-n}(V ; Z)$ onto the cohomology group $H^{n}(V ; Z)$, and if $V$ is nonorientable, then we take the coefficient group $Z_{2}$ and duality means the isomorphism of Poincaré-Veblen, i.e., the isomorphism of $H_{m-n}\left(V ; Z_{2}\right)$ onto $H^{n}\left(V ; Z_{2}\right)$.

(4) The cup-product is defined by the multiplication among integers. 
for cohomology classes $x, y$ in $H^{*}(K$; (j). If we put $y=1$, then the formula (4) gives us

$$
\phi^{*}(x)=p^{*} x \cup U_{n}
$$

for every class $x$ in $H^{*}\left(K\right.$; (अ). Also $\phi^{*}$ commutes with the Steenrod square operations.

The Stiefel-Whitney class $W_{j}$ of dimension $j(0 \leqq j \leqq n)$, is defined as the fundamental characteristic class of the associated $(j-1)$-sphere fiber space over the $j$-skeleton $K_{j}$ of $K$.

THEOREM 2. The Stiefel-Whitney class $W_{j}$ of the $(n-1)$-sphere fiber space $S$ satisfies the following formula,

$$
S q^{j} U_{n}=U_{n} \cup W_{j} \quad \text { for } 0 \leqq j \leqq n
$$

where $S q^{j}$ denotes the Steenrod square operation which raises dimension by $j$.

The proof of this theorem can be found in [2, Chap. II, Theorem II].

We remark that the relation (6) leads to the topological invariance of the Stiefel-Whitney classes of compact differentiable manifolds.

In the end of this section, we mention a property of the canonical isomorphism for double fiber structures. Let $A^{\prime \prime}$ be a fiber structure over $A^{\prime}$ with an open cell as fiber. $\phi^{\prime *}$ denotes the canonical isomorphism for $A^{\prime \prime}$ over $A^{\prime}$. Obviously, $A^{\prime \prime}$ is a fiber structure over $K$ with the open cell which is product cell of the fiber of $A^{\prime}$ over $K$ and that of $A^{\prime \prime}$ over $A^{\prime} . \phi^{\prime \prime *}$ denotes the isomorphism for $A^{\prime \prime}$ over $K$, then we have the relation

$$
\phi^{\prime *}=\phi^{\prime *} \phi^{*} \text {. }
$$

\section{Cup-products of realizable cohomology classes.}

Theorem 3. Suppose that $K$ is a finite cell complex and that $u_{1} \in H^{k_{1}}(K$; (S) and $u_{2} \in H^{k_{2}}\left(K\right.$; (j). If $u_{1}$ and $u_{2}$ are $O\left(k_{1}\right)$ - and $O\left(k_{2}\right)$-realizable, respectively, then the cup-product $\left({ }^{5}\right) u_{1} \cup u_{2}$ is $O\left(k_{1}+k_{2}\right)$-realizable.

Proof. By Definition 2, there exists two mappings, $f_{1}: K \rightarrow M\left(O\left(k_{1}\right)\right)$ and $f_{2}: K \rightarrow M\left(O\left(k_{2}\right)\right)$ having the following properties. For the sake of brevity, we use the notations $U_{1}, U_{2}$ instead of $U_{o\left(k_{1}\right)}, U_{o\left(k_{2}\right)}$. Then the relations,

$$
f_{1}^{*} U_{1}=u_{1}, \quad f_{2}^{*} U_{2}=u_{2}
$$

hold.

Now we shall define a mapping $\bar{H}$ of the product space $M\left(O\left(k_{1}\right)\right)$ $\times M\left(O\left(k_{2}\right)\right)$ into the cell complex $M\left(O\left(k_{1}+k_{2}\right)\right)$ as follows: $A_{O\left(k_{1}\right)} \times A_{O\left(k_{2}\right)}$ is a fiber space over the product space $B_{O\left(k_{1}\right)} \times B_{O\left(k_{2}\right)}$ with fiber, the $\left(k_{1}+k_{2}\right)$-cell $b_{1} \times b_{2}$ and with structural group, the orthogonal group $O\left(k_{1}+k_{2}\right)$. Since

(5) The cup-product is defined by the multiplication of the ring (5). Further, Theorem 3 holds generally in the case where the coefficient group is any ring. 
$O\left(k_{1}+k_{2}\right)$ contains the group $O\left(k_{1}\right) \times O\left(k_{2}\right)$, we can take the universal fiber space $A_{O\left(k_{1}+k_{2}\right)}$ over $B_{O\left(k_{1}+k_{2}\right)}$ and a classifying map $h$ of $B_{O\left(k_{1}\right)} \times B_{O\left(k_{2}\right)}$ into $B_{O\left(k_{1}+k_{2}\right)}$ which induces the fiber space $A_{O\left(k_{1}\right)} \times A_{O\left(k_{2}\right)}$ from $A_{O\left(k_{1}+k_{2}\right)}$. Let $H$ be the mapping of $A_{O\left(k_{1}\right)} \times A_{O\left(k_{2}\right)}$ into $A_{O\left(k_{1}+k_{2}\right)}$ induced by the classifying map $h$. Since $H$ maps the boundary of $A_{O\left(k_{1}\right)} \times A_{O\left(k_{2}\right)}$ into that of $A_{O\left(k_{1}+k_{2}\right)}, H$ defines a continuous mapping $\bar{H}$ of the product cell complex $M\left(O\left(k_{1}\right)\right) \times M\left(O\left(k_{2}\right)\right)$ into the complex $M\left(O\left(k_{1}+k_{2}\right)\right)$.

Let $j_{1}$ and $j_{2}$ be the projections of $M\left(O\left(k_{1}\right)\right) \times M\left(O\left(k_{2}\right)\right)$ onto $M\left(O\left(k_{1}\right)\right)$ and $M\left(O\left(k_{2}\right)\right)$ respectively, defined by the formulae,

$$
j_{1}(x, y)=x, \quad j_{2}(x, y)=y
$$

for each point $(x, y) \in M\left(O\left(k_{1}\right)\right) \times M\left(O\left(k_{2}\right)\right)$. Similarly, we define the projections $j_{1}^{\prime}$ and $j_{2}^{\prime}$ of $A_{O\left(k_{1}\right)}^{\prime} \times A_{O\left(k_{2}\right)}^{\prime}$ on to $A_{O\left(k_{1}\right)}^{\prime}$ and $A_{O\left(k_{2}\right)}^{\prime}$ respectively. Let $U_{1}^{\prime}$ and $U_{2}^{\prime}$ be the fundamental classes in the cohomology groups $H^{k_{1}}\left(A_{O\left(k_{1}\right)}^{\prime}\right.$; (S) and $H^{k_{2}}\left(A_{O\left(k_{2}\right)}^{\prime}\right.$; (S) $)$ which correspond naturally to $U_{1}$ and $U_{2}$.

We consider a cellular subdivision of the space $A_{O\left(k_{1}\right)}^{\prime}$ as follows: We can suppose a simplicial subdivision of the base space $B_{O\left(k_{1}\right)}$ which satisfies the condition that each simplex is contained in a coordinate neighborhood. We take product cells of such simplexes with the $k_{1}$-cell of the fiber. These cells make a cellular subdivision of $A_{O\left(k_{1}\right)}^{\prime}$. In the similar way, we can suppose such cellular subdivisions of the space $A_{O\left(k_{2}\right)}^{\prime}$ and of the space $A_{O\left(k_{1}+k_{2}\right)}^{\prime}$. Then we have:

LemMA 1. Under the above conditions, the relation

$$
H^{\prime *} U_{O\left(k_{1}+k_{2}\right)}^{\prime}=j_{1}^{\prime *} U_{1}^{\prime} \cup j_{2}^{\prime *} U_{2}^{\prime}
$$

holds, where $H^{\prime}$ is the mapping of $A_{O\left(k_{1}\right)}^{\prime} \times A_{O\left(k_{2}\right)}^{\prime}$ into $A_{O\left(k_{1}+k_{2}\right)}^{\prime}$ induced by $H$ and $U_{O\left(k_{3}+k_{2}\right)}^{\prime}$ is the fundamental class in $H^{k_{1}+k_{2}}\left(A_{O\left(k_{1}+k_{2}\right)}^{\prime}\right.$; (3) which correspond naturally to $U_{O\left(k_{1}+k_{2}\right)}$.

Proof. $A_{O\left(k_{1}\right)}^{\prime} \times A_{O\left(k_{2}\right)}^{\prime}$ has a double fiber structure, $A_{O\left(k_{1}\right)}^{\prime} \times B_{O\left(k_{2}\right)}$ over $B_{O\left(k_{1}\right)} \times B_{O\left(k_{2}\right)}$ and $A_{O\left(k_{1}\right)}^{\prime} \times A_{O\left(k_{2}\right)}^{\prime}$ over $A_{O\left(k_{1}\right)}^{\prime} \times B_{O\left(k_{2}\right)}^{\prime}$. Let $\phi_{1}^{*}, \phi_{2}^{*}$ denote the canonical isomorphisms for these fiber structures, respectively, and let $\phi^{*}$ denote that for $A_{O\left(k_{1}\right)}^{\prime} \times A_{O\left(k_{2}\right)}^{\prime}$ over $B_{O\left(k_{1}\right)} \times B_{O\left(k_{2}\right)}$. (7) gives us the relation,

$$
\phi^{*}=\phi_{2}^{*} \phi_{1}^{*} \text {. }
$$

By using the commutativity of (3), we have that $\left({ }^{6}\right)$

$$
\begin{aligned}
{H^{\prime}}^{*} U_{O\left(k_{1}+k_{2}\right)}^{\prime} & ={H^{\prime *}}_{\phi O\left(k_{1}+k_{2}\right)}^{*}\left(1_{O\left(k_{1}+k_{2}\right)}\right)=\phi^{*}(1) \\
& =\phi_{2}^{*} \phi_{1}^{*}(1)=\phi_{2}^{*}\left(j_{1}^{\prime *} U_{1}^{\prime}\right) \\
& =j_{1}^{\prime *} U_{1}^{\prime} \cup j_{2}^{\prime *} U_{2}^{\prime} .
\end{aligned}
$$

(6) For simplicity, we denote the canonical isomorphism $\phi_{O}^{*}{ }_{\left(k_{1}+k_{2},\right)_{n}}$ by $\phi_{O}{ }_{\left(k_{1}+k_{2}\right)}$, if $n=k_{1}+k_{2}$. 
Thus Lemma 1 has proved.

We have a diagram of the homomorphisms $\bar{H}^{*}, H^{\prime *}, j_{1}^{*} \cup j_{2}^{*}, j_{1}^{\prime *} \cup j_{2}^{\prime *}$ and if the isomorphisms of the cohomology groups induced by identifying the boundaries, $S_{O\left(k_{i}\right)}$ in $A_{O\left(k_{i}\right)}(i=1,2)$ and $S_{O\left(k_{1}+k_{2}\right)}$ in $A_{O\left(k_{1}+k_{2}\right)}$ to a point, respectively,

$$
\begin{aligned}
& H^{k_{1}}\left(A_{O\left(k_{1}\right.}^{\prime} \text {; (j) } \times H^{k_{2}}\left(A_{O\left(k_{2}\right)}^{\prime} \text {; (\$) }\right)=H^{k_{1}}\left(M\left(O\left(k_{1}\right)\right) ; \text { (S) } \times H^{k_{2}}\left(M\left(O\left(k_{2}\right)\right) \text {; (\$) }\right)\right.\right. \\
& j_{1}^{\prime *} \cup j_{2}^{\prime *} \downarrow \\
& H^{k_{1}+k_{2}}\left(A_{O\left(k_{1}\right)}^{\prime} \times A_{O\left(k_{2}\right)}^{\prime} \text {; (S) }\right) \\
& H^{*} \uparrow \\
& H^{k_{1}+k_{2}}\left(A_{O\left(k_{1}+k_{2}\right)}^{\prime} ; \text { (S) }\right) \\
& \downarrow j_{1}^{*} \cup j_{2}^{*} \\
& \rightarrow H^{k_{1}+k_{2}}\left(M\left(O\left(k_{1}\right)\right) \times M\left(O\left(k_{2}\right)\right) ;(\xi)\right. \\
& \uparrow \bar{H}^{*} \\
& =H^{k_{1}+k_{2}}\left(M\left(O\left(k_{1}+k_{2}\right)\right) ;(\mathfrak{S})\right. \text {, }
\end{aligned}
$$

where the horizontal arrow is a canonical homomorphism of cohomology groups which is induced by the inclusion mapping of pairs of spaces,

$$
\left(A_{O\left(k_{1}\right.}, \times A_{O\left(k_{2}\right)}, 0\right) \rightarrow\left(A_{O\left(k_{1}\right)} \times A_{O\left(k_{2}\right)},\left(A_{O\left(k_{1}\right)} \times A_{O\left(k_{2}\right)}\right)^{\cdot}\right) .
$$

Since the above diagram is commutative, the relation (8) leads to the formula,

$$
\bar{H}^{*} U_{O\left(k_{1}+k_{2}\right)}=j_{1}^{*} U_{1} \cup j_{2}^{*} U_{2},
$$

which plays an important roll in the proof of Theorem 3 .

Now we define a mapping $f_{1} * f_{2}$ from the cell complex $K$ into the product cell complex $M\left(O\left(k_{1}\right)\right) \times M\left(O\left(k_{2}\right)\right)$ by the formula,

$$
f_{1} * f_{2}(a)=f_{1}(a) \times f_{2}(a), \quad a \in K .
$$

Then this mapping induces a homomorphism $\left(f_{1} * f_{2}\right) *$ of the cohomology group

$$
H^{k_{1}+k_{2}}\left(M\left(O\left(k_{1}\right)\right) \times M\left(O\left(k_{2}\right)\right) ;(3)\right.
$$

into the cohomology group $H^{k_{1}+k_{2}}(K$; (H). Using the property that these induced homomorphisms and the cup-products are commutative, we have the relation,

$$
\begin{aligned}
\left(f_{1} * f_{2}\right)^{*}\left(j_{1}^{*} U_{1} \cup j_{2}^{*} U_{2}\right) & =f_{1}^{*} U_{1} \cup f_{2}^{*} U_{2} \\
& =u_{1} \cup u_{2},
\end{aligned}
$$

because it is easily seen that

$$
\begin{aligned}
& f_{1}=j_{1}\left(f_{1} * f_{2}\right), \\
& f_{2}=j_{2}\left(f_{1} * f_{2}\right) .
\end{aligned}
$$

We put $f=\bar{H}\left(f_{1} * f_{2}\right)$. This is a continuous mapping of $K$ into $M\left(O\left(k_{1}+k_{2}\right)\right)$. Combining the formulae $\left(8^{\prime}\right)$ and $(9)$, we obtain the relation, 


$$
\begin{aligned}
f^{*} U_{O\left(k_{1}+k_{2}\right)} & =\left(f_{1} * f_{2}\right)^{*} H^{*} U_{O\left(k_{1}+k_{2}\right)} \\
& =\left(f_{1} * f_{2}\right)^{*}\left(j_{1}^{*} U_{1} \cup j_{2}^{*} U_{2}\right) \\
& =u_{1} \cup u_{2} .
\end{aligned}
$$

The last formula (10) means that the class $u_{1} \cup u_{2}$ has an $O\left(k_{1}+k_{2}\right)$ realization.

4. Applications of Theorem 3. When the cell complex $K$ stated above is an $n$ dimensional differentiable manifold $V$ of class $C^{\infty}$, we can use Theorem 1 . Then we have the following result.

CoROllary 1. Let $V$ be an $n$ dimensional differentiable manifold of class $C^{\infty}$. Let $z_{1}, z_{2}$ be homology classes of respective dimension $n-k_{1}, n-k_{2}$ in $V$, where we suppose that $k_{1}, k_{2} \leqq n$. We take the integers or the integers modulo 2 as coefficients and take only the latter when $V$ is nonorientable. If the classes $z_{1}, z_{2}$ are realized by submanifolds, then their intersection class $z_{1} \cdot z_{2}$ is also realized by a submanifold.

Proof. The homology class $z_{1} \cdot z_{2}$ is dual to the cohomology class $u_{1} \cup u_{2}$ where the class $u_{i}$ is the dual of $z_{i}(i=1,2)(\mathrm{H}$. Whitney [6]). This fact together with Theorem 1 and Theorem 3 yield Corollary 1 .

Theorem 3 shows the existence of a mapping $f$ of $K$ into $M\left(O\left(k_{1}+k_{2}\right)\right)$. We state this fact as Corollary 2.

CoROLlary 2. If there exist continuous mappings $f_{1}, f_{2}$ of the cell complex $K$ into the cell complexes $M\left(O\left(k_{1}\right)\right)$ and $M\left(O\left(k_{2}\right)\right)$ which satisfy the condition that $f_{1}^{*} U_{1}=u_{1}, f_{2}^{*} U_{2}=u_{2}$. Then there exists a continuous mapping $f$ of $K$ into $M\left(O\left(k_{1}+k_{2}\right)\right)$ such that $f^{*} U_{O\left(k_{1}+k_{2}\right)}=u_{1} \cup u_{2}$.

So far we have proved that in a compact differentiable manifold the cupproduct of two realizable classes is realizable. In the next sections, we shall study the Steenrod square operations of realizable cohomology classes.

5. Squares of classes of $n$ dimension having $O(k)$-realization. In this section, we shall state theorems on the realizability of Steenrod square of $n$ dimensional cohomology classes which are realizable for $O(k) \subset O(n)$.

Now we denote by $V$ a compact differentiable manifold of dimension $m+n$ and denote by $W$ a submanifold in $V$ of dimension $m$. Let $z \in H_{m}\left(V ; Z_{2}\right)$ be the homology class defined by $W$ and $u \in H^{n}\left(V ; Z_{2}\right)$ be the cohomology class which is dual to $z$. Then the following is the main theorem of this note.

THEOREM 4. If the fiber space $N(W)$ of normal vectors over the submanifold $W$ in $V$ has a field of $(n-k)$-linearly independent vectors where we suppose that $k \leqq n$, then the square $S q^{k}(u)$ of the class $u$ can be also realized.

The condition of Theorem 4 is satisfied if and only if the fiber space $N(W)$ has $O(k)$ as its structural group. So, we can state the above fact simply as follows: 
THEOREM 5. If $u \in H^{n}\left(V ; Z_{2}\right)$ is realizable for $O(k) \subset O(n)$, then $S q^{k}(u)$ can be also realized.

REMARK. If the Stiefel-Whitney class $W_{k} \in H^{k}\left(W ; Z_{2}\right)$ is realizable, then Theorem 5 follows from Theorem 3 immediately. In the following, however, we shall give the proof without the fact.

In order to prove Theorem 5 , we state some preliminary results. By Theorem 1 there exists a mapping $f$ of $V$ into $M(O(k), n)$ such that the homomorphism $f^{*}$ of $H^{n}\left(M(O(k), n) ; Z_{2}\right)$ into $H^{n}\left(V ; Z_{2}\right)$ induced by $f$ satisfies the condition that

$$
u=f^{*} U_{o(k), n} .
$$

Next we consider the structure of the fiber space $A_{O(k), n}$ over $B_{O(k)}$.

Lemma 2. The fiber structure $A_{O(k), n}$ decomposes into the product of a closed $(n-k)$-cell $\bar{b}_{n-k}$ and $A_{o(k)}$.

Proof. We can choose a system of coordinate transformations for the fiber space $A_{O(k), n}$, any transformation of which leaves certain $n-k$ coordinates fixed in the fiber, a closed $n$-cell $b_{n}$.

6. The proof of Theorem 5. By Lemma 2 we can define a mapping of $A_{O(k), n}$ onto $A_{O(k)}$ which collapses the closed $(n-k)$-cell of fiber into a point. Let $q$ be such a map. On the other hand, we denote the identity mapping of $A_{O(k), n}$ onto itself by $\alpha$. We can define a mapping $\alpha \oplus q$ of $A_{O(k), n}$ into the Whitney sum $A_{O(k), n} \oplus A_{O(k)}$ (see Wu [7]) in such a way that

$$
\alpha \oplus q(x)=(\alpha(x), q(x)),
$$

for each point $x$ of $A_{O(k), n} . A_{O(k), n} \oplus A_{O(k)}$ is a fiber space over $B_{O(k)}$ with $b_{n} \times b_{k}$ as its fiber. Let $H$ denote a fiber mapping of $A_{O(k), n} \oplus A_{O_{(k)}}$ into the universal fiber space over $A_{O(n+k)}$ which is induced by a classifying map. We denote by $M$ the Whitney sum $A_{O(k), n} \oplus A_{O(k)}$ the boundary of which is identified into a point. Let $\bar{H}$ be the mapping of $M$ into $M(O(n+k))$ induced by $H$. Since $\alpha \oplus q$ maps $S_{O(k), n}$ in to the boundary $\left(A_{O(k), n} \oplus A_{O(k)}\right)^{\cdot}$, it induces a mapping $[\alpha \oplus q]$ of $M(O(k)), n)$ in to $M$. Then we get the following diagram of mappings of spaces,

$$
\begin{array}{rlrl}
A_{o(k), n} & \stackrel{\alpha \oplus q}{\longrightarrow} A_{o(k), n} \oplus A_{O(k)} \stackrel{H}{\longrightarrow} A_{o(n+k)} \\
& \stackrel{i \downarrow}{\rightarrow} M(O(k), n) \stackrel{[\alpha \oplus q]}{i \downarrow} M & \stackrel{\bar{H}}{\longrightarrow} M(O(n+k))
\end{array}
$$

where the vertical arrow $i$ shows the respective identifying map. In each square, commutativity holds.

The diagram (12) induces the following diagram of homomorphisms of cohomology groups and commutativity holds in each square; 


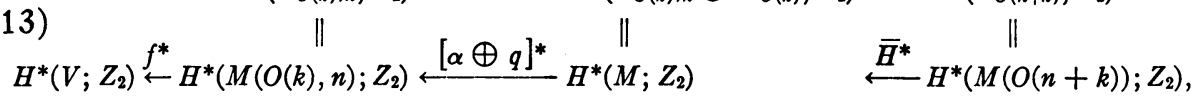

where $H^{\prime}$ is the mapping induced by $H$.

Let $U_{i}^{\prime}, i=k, n, n+k$, be the fundamental classes of $A_{O_{(k)},}^{\prime}, A_{O(k), n}^{\prime}$, $A_{O(n+k)}^{\prime}$ respectively and let $U_{i}$ be the fundamental classes of $M(O(k))$, $M(O(k), n), M(O(n+k))$ which correspond canonically to $U_{i}$ respectively. Let $j_{1}^{\prime}, j_{2}^{\prime}$ denote the projections of $A_{O_{(k), n}}^{\prime} \oplus A_{O(k)}^{\prime}$ onto $A_{O_{(k), n}}^{\prime}$ and onto $A_{O_{(k)}}^{\prime}$ respectively.

By the same argument as Lemma 1, we have the relation,

$$
{H^{\prime *}}^{\prime} U_{n+k}^{\prime}=j_{1}^{\prime *} U_{n}^{\prime} \cup j_{2}^{* *} U_{k}^{\prime}
$$

From definitions of $\alpha \oplus q$ and of $j_{i}^{\prime}$, we get the formulae,

$$
\begin{aligned}
& j_{1}^{\prime}(\alpha \oplus q)=\alpha, \\
& j_{2}^{\prime}(\alpha \oplus q)=q .
\end{aligned}
$$

They lead to the relations,

$$
\begin{aligned}
& (\alpha \oplus q)^{* j_{1}^{* *}}=\text { the identity isomorphism, } \\
& (\alpha \oplus q)^{*} j_{2}^{* *}=q^{*} .
\end{aligned}
$$

Using these formulae together with (14), we obtain the result,

$$
\begin{aligned}
(\alpha \oplus q){ }^{*}{H^{\prime *}}^{*} U_{n+k}^{\prime} & =(\alpha \oplus q)^{*}\left(j_{1}^{*} U_{n}^{\prime} \cup j_{2}^{\prime *} U_{k}^{\prime}\right) \\
& =(\alpha \oplus q)^{*} j_{1}^{*} U_{n}^{\prime} \cup(\alpha \oplus q)^{*} j_{2}^{* *} U_{k}^{\prime} \\
& =U_{n}^{\prime} \cup q^{*} U_{k}^{\prime} .
\end{aligned}
$$

By definition of the Stiefel-Whitney class of dimension $k$, we have $W_{k}$ $=\beta^{*} q^{*} U_{k}^{\prime}$. Hence we obtain the following,

$$
\begin{aligned}
U_{n}^{\prime} \cup q^{*} U_{k}^{\prime} & =U_{n}^{\prime} \cup \beta^{*} q^{*} U_{k}^{\prime} \\
& =U_{n}^{\prime} \cup W_{k} \\
& =S q^{k} U_{n}^{\prime} .
\end{aligned}
$$

We have, therefore, the relation

$$
(\alpha \oplus q)^{*} H^{\prime *} U_{n+k}^{\prime}=S q^{k} U_{n}^{\prime}
$$

(13) and (15) show that

$$
[\alpha \oplus q]^{*} \bar{H}^{*} U_{n+k}=S q^{k} U_{n},
$$


where

$$
V \stackrel{f}{\rightarrow} M(O(k), n) \stackrel{[\alpha+q]}{\longrightarrow} M \stackrel{\bar{H}}{\rightarrow} M(O(n+k)) .
$$

If we take the composite mapping $\bar{H}[\alpha \oplus q] f$ of $V$ into $M(O(n+k))$, we obtain, by (16), the result that

$$
\begin{aligned}
(\bar{H}[\alpha \oplus q] f)^{*} U_{n+k} & =f^{*}[\alpha \oplus q]^{*} \bar{H}^{*} U_{n+k} \\
& =f^{*} S q^{k} U_{n} \\
& =S q^{k} f^{*} U_{n} \\
& =S q^{k}(u) .
\end{aligned}
$$

Thus the result of Theorem 5 is completely proved.

7. A general result. Combining Theorem 3 and Theorem 5, we have a general result on the realizability of cohomology classes generated by cupproducts and Steenrod square operations of realizable classes:

THEOREM 6. Let $U_{i}, 0 \leqq i \leqq r$, be cohomology classes of dimension $n_{i}$ which are dual to homology classes determined by submanifolds in a compact differentiable manifold $V$. Suppose each $U_{i}$ is realizable for $O\left(k_{i}\right) \subset O\left(n_{i}\right)$. Then the cohomology class

$$
S q^{k_{1}} U_{1} \cup S q^{k_{2}} U_{2} \cup \cdots \cup S q^{k_{r}} U_{r}
$$

can be realized by a submanifold in $V$.

\section{REFERENCES}

1. R. Thom, Quelques propriêtés globales des variêtés differentiables, Comment. Math. Helv. vol. 28 (1954) pp. 17-86.

2. — Espace fibrés en spheres et carrés de Steenrod, Ann. École Norm. vol. 69(3) (1952) pp. 109-181.

3. N. E. Steenrod, Products of cocycles and extensions of mappings, Ann. of Math. vol. 48 (1947) pp. 290-320.

4. - Reduced powers of cohomology classes, Ann. of Math. vol. 56 (1952) pp. 47-67.

5. - The topology of fiber bundles, Princeton Mathematical Series 14, Princeton University Press, 1951.

6. H. Whitney, On products in complexes, Ann. of Math. vol. 39 (1938) pp. 397-432.

7. W. T. Wu, Sur les classes caractéristiques des structures fibrées sphériques, Publications de l'Institut de Mathématiques de l'Université de Strasbourg, Paris, Hermann et Cie, 1952.

TOHOKU University, SENDAI, JAPAN 